\title{
Application of Prenatal Health Education and Nutrition Intervention in Patients with Pregnancy-induced Hypertension
}

\author{
YuanPan ${ }^{1}$, Yang Yu and Ruizhi Liu ${ }^{\text {* }}$ \\ ${ }^{1}$ Reproductive medical center, First Hospital, Jilin University, Changchun city Jilin Province,130021 \\ adghrtgrd@163.com \\ *The corresponding author
}

Keywords: Prenatal, Health education, Nutritional intervention, Pregnancy-induced hypertension, Application

\begin{abstract}
Objective, to use prenatal health education and nutritional intervention strategies for pregnant women with pregnancy-related hypertension to explore the effect of interventions on patient outcomes and analyze them. Methods, a total of 90 pregnant women with gestational hypertension were selected and randomly divided into the observation group and the control group by random number method. The control group used the routine intervention nursing method. The observation group used prenatal health education and nutritional guidance. The values of blood pressure changes, delivery methods, weight changes, and other indicators were analyzed. Results Compared with the results of the control group, the normal rate of body weight gain during pregnancy in the observation group was significantly higher than that in the control group, and the difference between the groups was significant $(\mathrm{P}>0.05)$. In the observation group, the degree of satisfaction was significantly higher than that in the control group, with significant differences between groups. There was a statistically significant difference $(\mathrm{P}<0.05)$. The blood pressure of pregnant women in the observation group decreased significantly $(\mathrm{P}<0.05)$. Conclusion, The use of prenatal health education and nutritional intervention in patients with pregnancy-induced hypertension can help patients understand health knowledge, change their bad habits, and can help patients to improve clinical curative effect and improve the quality of life of patients. Maternal birth process progressed smoothly, effectively improving the outcome of childbirth, which is worth learning from in clinical practice.
\end{abstract}

Pregnancy-induced hypertension is a common and unique disease that affects the life and health of pregnant women and perinatal babies. It is also a common complication during maternity gestation. The current stage is the second cause of death from maternal deaths in China. The main clinical manifestations include high blood pressure, proteinuria, and edema, severe symptoms such as headache, coma, convulsions, and even maternal and child deaths. Prenatal examination and treatment awareness are enhanced, and maternal mortality caused by pregnancy-induced hypertension is greatly reduced ${ }^{[1-2]}$. Therefore, if the prevention and treatment are not treated in time or the method selection is not appropriate, and a series of more serious complications are caused, the condition will progress to severe pre-eclampsia or even eclampsia, threatening the life safety of the mother and child. The importance of obstetricians should be emphasized on this issue, and the prevalence of pregnancies in pregnant women is currently on a sharp upward trend. The disease is more frequent in the second trimester of pregnancy. It is easy to restrict the growth and development of fetuses with pregnancy-induced hypertension, cause maternal dystocia, or cause more severe birth injury, and the mortality rate shows a significant upward trend. According to relevant investigation reports, the main cause of the disease caused by the disease is the imbalance of nutritional adjustment in pregnant women and the lack of knowledge of health education during pregnancy ${ }^{[3]}$. At present, pregnancy-induced hypertension, as a gestational complication with high-risk characteristics, has undergone tremendous changes in people's lifestyle in recent years and has shifted back to child-bearing age. It has become an early diagnosis and treatment of complications of pregnancy and maternal complications and has improved quality. An important measure. In this article, prenatal health education and nutritional intervention in patients with pregnancy-induced hypertension were used to measure various growth indexes and their results 
were comprehensively analyzed.

\section{Clinical Data and Methods}

Information. In our hospital, 90 cases of pregnant women with hypertension were identified based on clinical data. The selected patients met the diagnostic criteria of the disease and were randomly assigned to the control group and the observation group after screening. There were 45 patients included in the observation group. The patients were between the ages of 28-39 years old with an average of $(34.4 \pm 4.3)$ years; the control group had 45 patients in the pregnancies group, and the ages of all the enrolled patients were between 26-41 years old. Between the average $(33.2 \pm 3.0)$ years old. All pregnant women were approved by the ethical committee of the hospital and in the informed consent bookmark. By comparing the general basic correlation data such as age, gestational age, heart rate, and clinical manifestation between the groups, the $\mathrm{P}$ value greater than 0.05 was not statistically significant and comparable. Inclusion criteria: The diagnosis of pregnancy-induced hypertension is consistent with its diagnostic criteria; good compliance; no other serious complications. Exclusion criteria: mental disorders, failure to successfully communicate; merger of liver and kidney failure, severe cardiovascular and cerebrovascular disease.

Interventions. Pregnant women in the control group used the hospital's regular care model. The observation group used prenatal health education and nutrition interventions and comprehensive nursing interventions, and specific measures for prenatal health education: confirmation of the needs of its health education, but paid attention to the protection of patient privacy, and at the same time obtained the absolute trust of patients, patiently informed patients and The purpose and related content of the family's education; according to the level of cultural knowledge of patients and their families and the ability to recognize things, formulate specific health education programs and measures [4], to guide patients to reasonably arrange treatment time according to their own work and life needs, and Emphasize the importance and necessity of treatment, and gain patient understanding and cooperation. The contents mainly include knowledge training for patients, through the promotion of relevant knowledge and health guidance consultation to enable patients to master, actively cooperate with the operation, help to complete the smooth inspection [5]. Observe the conditions of the patients admitted to the hospital in real time, monitor the development of the fetus and detailed records in a timely manner. Mental care in pregnancy makes pregnant women inevitably produce fear, anxiety and other negative emotions, coupled with greater psychological pressure, adverse conditions continue to adversely affect the production even worse. It is necessary to communicate with the patient several times for advice and guidance, or kind and friendly communication, so as to increase the trust of the patient and establish their own confidence. You can also use gentle language and friendly attitude to establish a good nurse-patient relationship with patients and their families as far as possible to achieve active and active participation. More emphasis is placed on using interpretative, encouraging, persuasive language to explain relevant knowledge to patients and clarify their necessity. Relieve tension and fear, which helps patients actively cooperate with treatment [6-7]. Detailed patience expresses the necessity of surgery and the safety of the surgical procedure. Maternal and family members raise relevant questions and give detailed patient explanations. The detailed explanations and practical actions that may be encountered during the surgery and the correct response methods can be enhanced. Maternal self-confidence, reduce anxiety and reduce maternal psychological burden. It also introduced the situation of doctors operating on the day and established doctors' prestige to increase the safety factor. Nutrition interventions: Instruct patients to have a clear and reasonable diet, and tailor diet plans for different individuals. Eat small quantities of food, eat more fruits and vegetables, and eat more foods rich in protein. Special attention should be paid to dietary intake of light, low-salt, low-fat, and low-cholesterol foods to keep the urine clear, avoid bloating, avoid cold, spicy, and irritating foods. . . If allowed to give dietary guidance according to the patient's preferences, but must maintain a balanced nutrition, to ensure adequate daily food intake, and enhance the patient's body resistance [8]. 
Inspection Methods. Take two sets of pregnant women during pregnancy weight gain, complications during pregnancy and delivery methods, etc. Pregnancy weight gain in normal pregnant women $=$ pre-delivery weight - pre-pregnancy weight, and recommended for appropriate weight gain during pregnancy based on the pre-pregnancy BMI. According to the Ministry of Health Hypertension efficacy evaluation criteria for assessing blood pressure changes. Significantly reduced diastolic blood pressure $>10 \mathrm{~mm} \mathrm{Hg}$ and decreased to normal range, or diastolic blood pressure decreased by $>20 \mathrm{~mm} \mathrm{Hg}$; effective diastolic blood pressure decreased by $<10 \mathrm{~mm} \mathrm{Hg}$, but has fallen to normal range, or decreased diastolic blood pressure by $10-20 \mathrm{mmHg}$; Or systolic blood pressure drop> $30 \mathrm{~mm} \mathrm{Hg}$; Invalid for the blood pressure does not meet the above effective criteria. Total efficiency $=($ effective + effective $) /$ total number of cases $\times 100 \%$.

Statistical Analysis. For the two groups treated by different means, the data obtained after the treatment were recorded by SPSS 14.0 statistical software, statistical analysis and comparison. If the measurement data is expressed as the mean \pm standard deviation $(x \pm s)$, the t-test is used; if the count data is expressed in terms of rate (\%), the $\mathrm{X} 2$ test is required. After analysis between groups, $\mathrm{P}<0.05$ showed significant difference between the groups with statistical significance.

\section{Results}

Weight Gain of Pregnant Women in Two Groups. According to the results in Table 1, it can be seen that the normal rate of body weight gain in the observation group was $88.9 \%$ compared with the control group. There was a significant difference between the groups $(\mathrm{P}<0.05)$, as shown in the Table 1.

Table 1 The weight increasing of pregnant women in two groups

\begin{tabular}{llll}
\hline Group & Low level $(\mathrm{n})$ & Normal level $(\mathrm{n})$ & High level $(\mathrm{n})$ \\
\hline Control group & $10(22.2 \%)$ & $24(53.3 \%)$ & $11(24.4 \%)$ \\
Observation group & $3(6.7 \%)$ & $40(88.9 \%)$ & $2(4.4 \%)$ \\
\hline
\end{tabular}

Blood Pressure Status after Treatment In both Groups. After treatment, the systolic blood pressure and diastolic blood pressure of the treatment group were significantly lower than those of the control group, and the blood pressure decreased significantly more than that of the conventional group. The difference was statistically significant $(\mathrm{P}<0.05)$.

Table 2 Blood pressure condition comparison after treatment of patients in two groups

\begin{tabular}{llll}
\hline Group & $\mathrm{n}$ & $\begin{array}{l}\text { Systolic blood pressure } \\
(\mathrm{mmHg})\end{array}$ & $\begin{array}{l}\text { Diastolic blood pressure } \\
(\mathrm{mmHg})\end{array}$ \\
\hline $\begin{array}{l}\text { Observation } \\
\text { group }\end{array}$ & 45 & $134 \pm 3$ & $83 \pm 2$ \\
Control group & 45 & $148 \pm 5$ & $95 \pm 1$ \\
\hline
\end{tabular}

The Antihypertensive Effect of the Two Groups after Treatment. The total effective rate of hypertension after intervention was significantly higher than that of the control group $(\mathrm{P}<0.05)$, as shown in Table 2.

Table 3 The comparison of depressed pressure of patents in two groups

\begin{tabular}{lllllll}
\hline Group & $\mathrm{n}$ & Obvious effect $(\mathrm{n})$ & effect $(\mathrm{n})$ & $\begin{array}{c}\text { Invalid } \\
(\mathrm{n})\end{array}$ & $\begin{array}{c}\text { Total } \\
(\%)\end{array}$ & effect \\
\hline $\begin{array}{l}\text { Observation } \\
\text { group }\end{array}$ & 45 & 27 & 16 & 2 & 95.6 \\
Control group & 45 & 21 & 12 & 12 & 73.3 \\
\hline
\end{tabular}


Evaluation Satisfaction. The satisfaction of the two groups of patients was evaluated after the intervention of health instruction. The results are shown in Table 2. The satisfaction of the patients in the observation group reached $933.3 \%$; the satisfaction of the control group was only $71.1 \%$, and the satisfaction of the observation group increased. $22.2 \%$, there was a large difference in satisfaction rate between groups, and there was significant difference between the analyzed groups $(\mathrm{P}<0.05)$.

Table 4 the survey results of satisfaction

\begin{tabular}{llllll}
\hline Group & $\mathrm{n}$ & Good & Normal & Unsatisfaction & satisfaction (\%) \\
\hline $\begin{array}{l}\text { Observation } \\
\text { group }\end{array}$ & 45 & 29 & 13 & 3 & 93.3 \\
Control group & 45 & 20 & 12 & 13 & 71.1 \\
\hline
\end{tabular}

\section{Conclusion}

Clinical health and nutrition interventions can effectively prevent adverse effects on pregnant women with high blood pressure due to lack of knowledge and lack of communication among medical staff. And professional clinical intervention can guarantee certain nursing care in the occurrence, development and prognosis of the disease. It can also further simplify the patient's medical treatment process, reduce the incidence of complications, reduce the cost of medical care, and successfully make pregnant women produce. In addition, the standardized training of nursing staff's psychological nursing knowledge should be given as timely, effective, and correct health education as possible, so as to enhance treatment compliance and ensure that the nursing effect is maximized. Therefore, clinical interventions can help patients recover their health, improve patient comfort, and improve their prognosis. They can also increase patient satisfaction with treatment and increase patient satisfaction with care, reduce adverse effects, and improve the quality of life of pregnant women. Good results, reduce the degree of harm, especially play a positive role in maternal hypertension treatment, which helps to ensure the safety of mother and child.

The results of this study show that the use of prenatal health education and nutrition guidance methods compared with the results of the control group, the normal rate of body weight gain during pregnancy in the observation group was significantly higher than the control group, the difference between the groups was significant $(\mathrm{P}>0.05)$; Satisfaction was significantly higher than that of the control group, with significant differences between the groups $(\mathrm{P}<0.05)$. Pregnant women in the observation group had significantly lower blood pressure during pregnancy $(\mathrm{P}<0.05)$. It shows that nursing intervention is the key link in the recovery of maternal hypertension treatment and prepares the corresponding care for the smooth production of pregnant women. Therefore, according to the etiology and characteristics of patients, they must also pay attention to the needs of clinical patients for their health knowledge in the stage of clinical treatment, to ensure the physical and mental health of patients, increase comfort, and promote recovery of patients. Based on the clinical treatment of the disease, this study will further carry out the work related to health education for the patient, accumulate more experience for clinical work in the future, and better serve the patient.

In summary, in the treatment of hypertensive pregnant women not only need to choose the appropriate treatment and in the treatment of the same time need to take relevant knowledge and education activities, after the implementation of the patient to achieve effective relief of adverse psychological mood, improve the patient's clinical symptoms, and ultimately Smooth production, therefore, has a positive guiding significance in the clinical worthy of further reference.

\section{References}

[1] Jiang Songmei, Yao Xuewei, Mao Hongying. Effects of Individualized Nutrition Guidance on Pregnancy Outcomes during Pregnancy [J]. Chinese Women and Child Health Research, 2015, 26 (3): 619-620.

[2] Chu Zhou, Tao Fangbiao, Hao Jiahu, et al. Relationship between pre-pregnancy body mass 
index and 4 adverse pregnancy outcomes [J]. Chinese Public Health, 2012, 28 (6): 763-766.

[3] Tang Huiyan, Xing Fengmei, Gong Sufen. Research progress in the effect of prenatal health education and exercise during pregnancy on the delivery process[J].Maternal and Child Health Care of China,2012,10(10):1597-1600.

[4] Surabhi N, George N, Desislava M, et al. Maternal serum retinol-binding protein-4 at 11-13 weeks'gestation in normal and pathological pregnancies [J]. Metabol, 2013, 62 (6): 814-819.

[5] Jiang Yajun. Discussion on the relationship between prenatal antenatal health education and pregnancy outcomes [J]. Chinese maternity and child care, 2012, 32(14):5052-5053.

[6] Chung JG, Taylor RS, Thompson J M, et al. Gestational weight gain and adverse pregnancy outcomes in a nulliparous cohort [J]. Eur JObstet Gynecol Reprod Biol, 2013, 167(2): 149-153.

[7] Fruscalzo A, Londero AP, Driul L, et al. First trimester concentrations of the TTR-RBP4-retinol complex components as early marker of insulin-treated gestational diabetes mellitus: Clinical Chemistry and Laboratory Medicine (CCLM) [J]. Health \& Social Work, 2015, 4 (4) : 91-116.

[8] Liu Y, Dai W, Dai X, et al. Prepregnancy body mass index andgestational w eight gain w ith the outcome of pregnancy: a 13-yearstudy of 292,568 cases in China[J]. Arch Gynecol Obstet, 2012, 286 ( 4) : 905-911.

[9] Zhang Yanyan. Nursing Care of Patients with Pregnancy-induced Hypertension Syndrome [J].Chinese Health Nutrition: mid-term, 2014, 24(4):162.

[10] Guo Yanping, Cong Huiting. Nursing of hypertensive disorders during pregnancy [J]. The latest medical information in the world: Electronic publishing, 2014(19): 219.

[11]Jiang Ying, Ding Hui. Evaluation of the effect of personalized nutritional guidance on pregnancy health and pregnancy outcomes $[\mathrm{J}]$. Chinese Journal of Woman and Child Health Research, 2016, 27(5):600-605. 